Instituto Internacional de Investigación y Desarrollo Tecnológico Educativo INDTEC, C.A.

DOI: https://doi.org/10.29394/scientific.issn.2542-2987.2017.2.6.1.10-28

OAI-PMH: http://www.indteca.com/ojs/index.php/Revista Scientific/oai

\title{
Formación Docente en Técnicas de Superaprendizaje Aplicadas a la Enseñanza de la Matemática en la Educación Secundaria
}

\author{
Autor: Avilner Rafael Páez Pereira \\ Universidad Nacional Experimental "Rafael María Baralt", UNERMB \\ profavilnerp@gmail.com \\ Zulia, Venezuela
}

\section{Resumen}

El propósito del estudio fue formar al docente del LB "José Véliz" para el proceso de enseñanza de la matemática mediante la aplicación de técnicas de superaprendizaje, fundamentado en la modalidad Investigación Acción Participativa, propuesta por López de Ceballos, (2008), siguiendo el modelo de los ciclos de acción de Lewin (1946), citado por Latorre (2007), sustentado en las teorías del humanismo, Martínez (2009); las inteligencia múltiples, Armstrong (2006); el Superaprendizaje de Sambrano y Stainer, (2003). Enmarcado dentro del paradigma Crítico - Social, en el tipo Investigación Cualitativa se hizo un plan de acercamiento al grupo, donde mediante lluvia de ideas y entrevistas informales se listaron los principales problemas los cuales fueron jerarquizados para luego llevar a cabo un proceso de sensibilización y la formulación de un plan general de acción. Entre los resultados se realizaron 6 talleres de formación sobre técnicas de respiración, relajamiento, aromaterapia, musicoterapia, programación positiva, color en el aula, la canción en los algoritmos matemáticos, en los cuales se establecieron procesos de reflexión acerca de los beneficios u obstáculos obtenidos en la aplicación de éstas en la transformación de la realidad educativa, elaborándose una estrategia didáctica producto de las experiencias alcanzadas.

Palabras clave: superaprendizaje; matemática; formación. 


\title{
Teacher Formation in Super Learning Techniques Applied to the Teaching of the Mathematic in the Education Secondary
}

\begin{abstract}
The purpose of the study was to train LB "José Véliz" teacher for the teaching of mathematics through the application of super-learning techniques, based on the Research Participatory Action modality, proposed by López de Ceballos, (2008), following the model of the Lewin cycles of action (1946), quoted by Latorre (2007), based on the theories of humanism, Martínez (2009); multiple intelligence, Armstrong (2006); the Super learning of Sambrano and Stainer, (2003). Within the framework of the Critical - Social paradigm, in the type Qualitative Research, a plan of approach to the group was made, where through brainstorming and informal interviews the main problems were listed, which were hierarchized and then carried out an awareness - raising process. formulation of an overall plan of action. Among the results were 6 training workshops on techniques of breathing, relaxation, aromatherapy, music therapy, positive programming, color in the classroom, song in mathematical algorithms, in which processes of reflection were established on the benefits or obstacles obtained in the application of these in the transformation of the educational reality, elaborating a didactic strategy product of the experiences reached.
\end{abstract}

Keywords: super learning; mathematic; formation. 


\section{Introducción}

Actualmente, la preparación de los docentes para tomar parte activa en las investigaciones que se desarrollan, adquiere cada día una mayor significación, por lo cual, resulta imprescindible que los mismos no sólo sean capaces de conocer, identificar, y manejar adecuadamente todas las técnicas y métodos del cual se pueden servir para dar cumplimiento a los objetivos que han trazado para sus trabajos, sino tener tiempo y motivación para trabajar y transformar sus entornos.

En ese orden de ideas, el propósito del siguiente estudio consiste en formar al docente de la institución LB "José Véliz" mediante procesos de participación crítica y reflexiva, en el dominio de técnicas innovadoras de superaprendizaje que puedan ser aplicadas en la enseñanza de diversos métodos matemáticos rompiendo las diferentes barreras que obstaculizan el aprendizaje de la asignatura por parte de los estudiantes, considerando el proceso investigativo como el medio que permite generar cambios en la realidad social y educativa que se desarrolla en la institución mediante la constante formación académica de los profesionales en ejercicio.

En conformidad con lo anterior, la preocupación del personal docente que labora en la institución LB "José Véliz" en el área de la matemática e incluso en las otras disciplinas que se valen de la asignatura, debe estar orientada a la búsqueda de alternativas innovadoras que rompan las diferentes barreras que obstaculizan el aprendizaje y estimulen de una u otra manera el interés y el aumento del rendimiento académico en la mencionada disciplina y contribuir en la transformación de la realidad a través de la formación permanente.

\section{Percepción del investigador}

En el Liceo Bolivariano (LB) José Véliz, ubicado en la comunidad de las Cocuizas, parroquia Montaña Verde, municipio Torres del estado Lara, los 
estudiantes manifiestan poco interés por la resolución de ejercicios matemáticos, y para muchos la asignatura es considerada difícil, aburrida, innecesaria y la razón por la cual no puedan avanzar en sus estudios, sin dejar de mencionar el temor que la disciplina genera en los mismos incluso antes de ingresar a la institución o al enfrentarse a un nuevo profesor al año escolar siguiente.

En ese sentido, uno de los problemas que se denota comúnmente en los ambientes de clase, no siendo la excepción en el LB "José Véliz", es que los profesores de las otras disciplinas que requieren de las matemáticas como herramienta, al parecer no sitúan ni interrelacionan adecuadamente las ideas y conceptos centrales de esta asignatura, puesto que en su formación han aprendido a eludir el uso de esta materia y dicha actitud mantienen, a pesar de que en sus actividades académicas, las matemáticas cada día cobran mayor relevancia, por lo cual el interés de los estudiantes por las matemáticas quizás tenga que surgir necesariamente de la misma materia y no de la interacción con las otras ciencias.

De este modo, es probable que un gran número de factores sigan contribuyendo a que esta situación no cambie debido a que según lo observado con frecuencia, el maestro está acostumbrado a este estado de cosas y lo ve como natural; primero, por lo extenso de los programas, donde el mismo decide cubrirlos en su totalidad y no se da tiempo para generar el diálogo, fomentar las intervenciones de los aprendices y hacerles ver que es posible sacar más provecho a los tiempos de las actividades; y segundo porque ve obligatorio en el momento de impartir sus clases, la necesidad de ser conductista en su labor por la diversidad de métodos que se enseñan, según su perspectiva, mejor mediante este tipo de metodología de enseñanza.

En continuidad con lo anterior, en el LB "José Véliz", se observa una necesidad en la propuesta de nuevas formas de enseñanza, donde se promuevan aprendizajes significativos en los estudiantes, considerando 
además que el contexto donde funciona la institución carece de una adecuada infraestructura e incluso la misma se encuentra en dos espacios gestionados por las comunidades y el personal del plantel. Una ubicada en las instalaciones de PEDECA, sector las "Cocuizas" y la otra en la Iglesia Nuestra Sra. de Fátima en el Sector "Morroco", las cuales están regularmente acorde para el funcionamiento, aunque con muchas deficiencias en cuanto a las condiciones que debe reunir para funcionar como plantel educativo.

\section{Referentes Teóricos}

\subsection{Investigación Acción Participante}

Según López de Ceballos, (2008: 37), el método de la investigación acción participación (IAP) se basa en la mezcla de dos procesos, uno relacionado al conocimiento y el otro a la acción, pero en ambos se involucra a los sujetos cuya realidad se aborda, sin dejar de mencionar que se busca la conexión entre la teoría y la práctica para crear las mejores condiciones para el aprendizaje, así como se impulsa la toma de conciencia de una comunidad en específico sobre su propia realidad, y que le permita a sus miembros emprender un conjunto de acciones transformadoras aprovechando los recursos con los que cuentan.

En ese sentido, según la autora antes mencionada, cuando se realiza una investigación acción participante, se generan tres componentes fundamentales que se complementan entre si y por lo tanto, se puede asegurar lo siguiente:

- La investigación es vista como un procedimiento crítico-reflexivo, sistemático y controlado que sirve para estudiar uno o varios fenómenos de la realidad desde una perspectiva práctica.

- La acción no se considera el objetivo principal del proceso investigativo, pero a través de ésta se recogen datos y se genera conocimiento, al 
mismo tiempo que se convierte en una forma de intervenir en una determinada situación.

- La participación no es exclusiva de los investigadores profesionales, sino que el papel protagónico lo tiene la comunidad en la cual se desarrolla la acción, donde los sujetos que la conforman no son considerados como simples objetos de estudio sino como entes activos dedicados a indagar, construir y transformar su entorno.

\subsection{Teoría Humanista del Aprendizaje.}

En el proceso investigativo que se desarrolla es importante destacar el gran valor que tienen las habilidades y virtudes humanas incluso hasta las que se creen son las más simples, como dirigir la atención hacia lo que se considera bello, así como a las capacidades afectivas del ser humano. Es por ello que enseñar a apreciar las cualidades propias, y las del prójimo, constituye un aspecto relevante para el desarrollo integral de un individuo, partiendo de las cosas que le parecen interesante al estudiante y asociándolas con las que necesita conocer, por lo que el estudio del proceso de aprendizaje desde el punto de vista humanista se considera una base fundamental en esta investigación.

En ese orden de ideas, según Martínez (2009: 15), dentro del aprendizaje humanista se encuentra el denominado aprendizaje experiencial, que según el autor es una poderosa metodología del aprendizaje, dirigida a la formación y transformación de las personas como individuos en una íntima interrelación con otros individuos en la conformación de equipos, para alcanzar el desarrollo de sus competencias, así como el fortalecimiento de sus valores, tomando en cuenta que las personas viven sensaciones, emociones, situaciones y aprenden de ellas, actuando estas experiencias como fijadores del aprendizaje. 
Las características anteriores, constituyen el punto de partida para formar en los docentes del LB "José Véliz", un proceso de enseñanza aprendizaje donde se considere el mundo que envuelve al estudiante, las emociones, experiencias de vida y la percepción que tiene el educando de la realidad educativa y de las diversas sensaciones y estímulos que recibe del entorno, entendiendo además que la formación es la preparación idónea del personal dedicado al proceso educativo, permitiéndole actualizar y ampliar los conocimientos construidos antes y durante su labor diaria..

En conformidad con lo anterior, Rogers (2002), (citado en Martínez, 2009: 38), expresa que el individuo es el único que puede conocer por completo su campo de experiencia. El autor reconoce que no siempre es fácil entender la conducta desde el marco de referencia interno de otra persona, puesto que se está limitado a la percepción consciente y comunicación de experiencias de cada individuo. No obstante, un entendimiento empático de las experiencias de una persona siempre será útil estudiarla para de esta forma comprender los procesos de personalidad.

Entonces, en referencia al autor anterior, la conducta supone una satisfacción de las necesidades tal como éstas son percibidas, más que el estímulo o realidad objetiva, y dando como ejemplo el hecho de que un bebé puede ser tomado en brazos por una persona afectuosa, pero si su percepción de esta situación constituye una experiencia extraña o aterradora, es esta percepción la que influirá en su comportamiento, dicha situación puede extrapolarse al ambiente de clase en cuanto a la predisposición que puedan tener los estudiantes sobre las formas y métodos utilizados por el docente para la enseñanza de la matemática.

\subsection{Teoría de las Inteligencias Múltiples}

La teoría de las inteligencias múltiples según Armstrong (2006: 36), proporciona una postura optimista en cuanto a las mejoras de las 
competencias de los estudiantes a través de la estimulación adecuada en los periodos críticos de aprendizaje, considerando que toda persona posee habilidades nucleares y varias maneras de ser inteligente en cada una de las inteligencias existentes, y aunque a excepción de casos críticos, todos los individuos tienen la capacidad de desarrollar las diferentes inteligencias hasta un nivel razonable de competencia si reciben la motivación precisa.

De este modo, aplicando esta teoría en el aula, según el autor anterior, se entiende que no se tiene un número de individuos en clase, sino un número mucho de mayor de potenciales a desarrollar, sin dejar de mencionar que lo común es que cada individuo alcance un alto nivel de desarrollo en unas inteligencias y un nivel algo más bajo en otras, o expresado de otra manera, existen en cada ser áreas fuertes y áreas débiles de aptitudes.

En referencia a lo descrito, de acuerdo con Armstrong (2006: 40), en el cerebro humano existen distintas regiones que generan diferentes formas de asimilar y acomodar los procesos mentales, lo cual influye en un modo específico en el procesamiento de las informaciones que se perciben a través de los sentidos, así como también en el desarrollo de ciertas competencias.

Entonces, para el autor anterior, cada zona del cerebro asume un tipo de solución a un problema particular que se presente, o desarrolla una habilidad físico-cognitiva de acuerdo a las demandas del entorno cultural donde se desenvuelva el individuo, y es de allí de donde se desprenden las diversas maneras de comprender la inteligencia, rompiendo de este modo, con la idea preconcebida de una inteligencia que apunta a una sola dirección.

En ese sentido, en el trabajo de investigación que se desarrolló se tomaron los planteamientos de Armstrong (2006), debido a que se busca la formación de los docentes del LB "José Véliz" en el desarrollo de capacidades como pensar, observar, colaborar, compartir, representar y sentir a través de unos contenidos, y no a la asimilación pura de los mismos, donde se deben percibir las experiencias como un todo contextualizado, y de acuerdo a un eje 
temático en torno al cual se organicen las actividades, contemplando además el uso de temas relacionados con la inteligencia musical, cuyo factor resulta importante en la fundamentación y aplicación de técnicas de supreaprendizaje.

\subsection{El Superaprendizaje}

Esta teoría representa la base de la investigación que se realiza, puesto que tiene como propósito formar al docente en el proceso de enseñanza aprendizaje de la matemática mediante la aplicación de técnicas de superaprendizaje, considerando que en la educación venezolana puede ser vista como una metodología de vanguardia con la capacidad de revolucionar los métodos de enseñanza en todo el sistema educativo, debido a que rompe con el esquema tradicional de instrucción y se plantean estrategias innovadoras para mejorar el rendimiento en áreas críticas como lo ha sido la matemática.

En conformidad con lo anterior, el superaprendizaje es la obtención de resultados superiores en la construcción del conocimiento mediante el uso de diversos recursos para la estimulación multisensorial, permitiendo al sujeto la asimilación más fácil y eficiente del mimo, así como, su retención durante mayor tiempo en la memoria.

Al respecto, Sambrano y Stainer, (2003), (citados en Álamo y Moronta, 2007: 66), definen el superarendizaje como la adquisición acelerada de conocimientos, fluidamente y sin estrés, tratándose de un proceso de aprendizaje basado en una presentación sensorial rica, donde, por medio de canciones, dramatizaciones, juegos, imaginación, diálogos, roles, entre otros, se cumplen los objetivos de un contenido, de una manera divertida y eficaz al mismo tiempo, demostrando resultados muy superiores en términos del aprendizaje producido, en comparación con otras técnicas, ya que permite la asimilación más fácil, rápida y efectiva del conocimiento, así como, su retención durante mayor tiempo en la memoria y la aplicación inmediata. 
En referencia a lo descrito, el superaprendizaje según los autores antes mencionados involucra aprender a aprender con todo el cerebro, donde las cosas que se tienen que asimilar se deben sentir como una necesidad fisiológica, quererlas y, al mismo tiempo, entenderlas, lo cual promueve en los participantes la producción activa del conocimiento más que recepción pasiva, puesto que bajo este método se crean ambientes emocionalmente positivos, creativos y divertidos, que dejan huellas en los educandos, utilizando y aprendiendo con todo el cerebro, empleándolo a plenitud.

En ese sentido, en la investigación que se desarrolla se hará uso de las diversas técnicas propuestas con el fin de instruir a los docentes del área de las matemáticas y demás ciencias exactas del LB “José Véliz" en la aplicación de las mismas para mejorar el proceso de enseñanza aprendizaje en los estudiantes de la institución.

En ese orden de ideas, Sambrano y Stainer, (2003), (citados en Álamo y Moronta, 2007: 67), manifiestan que entre las estrategias de superaprendizaje más comunes se encuentran el estado de relajación, el ritmo sincronizado, la respiración rítmica, la música, la aromaterapia, los colores, el clima en el aula y la comunicación consciente asertiva, las cuales serán utilizadas en la consecución de los objetivos que se pretenden en la investigación e incrementar el desarrollo del proceso de enseñanza aprendizaje de la matemática del plantel LB "José Véliz" mediante talleres de formación para el docente.

\subsection{Aprendizaje de las matemáticas}

Se entiende que la matemática representa una de las ciencias más utilizada en el mundo, constituida por leyes y reglas que no se pueden cambiar, donde se establecen relaciones entre cantidades, magnitudes y propiedades, así como las operaciones lógicas para trabajar con las mismas, siendo 
fundamental en el ámbito educativo por ser además la base para otras ciencias como la química y la física.

Por otra parte, el uso de la matemática va mucho más allá de una simple disciplina académica, y se incluye en prácticamente todas las ramas del saber humano puesto que se recurre a modelos matemáticos para los trabajos de ingeniería, en las tecnologías más avanzadas, como las de los vuelos espaciales, de las modernas técnicas de diagnóstico médico, en la meteorología y en los estudios financieros por nombrar algunos.

En el mismo orden de ideas, Bronzina y otros (2009: 16) manifiestan que en la actualidad, en cuanto a la matemática, el énfasis está puesto en que los estudiantes tengan la posibilidad de interpretar datos, establecer relaciones y patrones de cambio, poner en juego conceptos matemáticos, analizar regularidades y la razonabilidad de resultados, planificar estrategias de solución, ensayar procedimientos y aceptarlos o descartarlos, registrar procedimientos utilizados, argumentar y defender posiciones propias del educando.

Igualmente, los autores mencionados expresan que dentro de los dominios contenidos que deben alcanzar los estudiantes en su etapa de escolaridad se encuentran cinco bloques principales que son:

1). Numérico: referente al manejo de los números y las diversas operaciones derivadas. 2). Geométrico: el cual se relaciona con la comprensión del espacio y la forma de los objetos. 3). De la medición: referido a la aprehensión de nociones de tamaños y medidas. 4). Estadístico: en el cual se estudia y se trata la información a través de tablas y gráficos. 5). Variacional: vinculado al estudio del cambio y al reconocimiento de regularidades y patrones observables en un problema 0 situación. (Bronzina y otros, 2009: 17)

Asimismo, Santaolalla (2009: 11), en consonancia con la forma en que se concibe el aprendizaje matemático, señala que en su mayoría este se ha 
manejado desde una concepción estructuralista, especialmente cuando se refiere al aprendizaje de conceptos, donde se supone que aprender es alterar estructuras, y que estas alteraciones se realizan de manera global y por ello es que la matemática es una ciencia que a nivel educativo representa una asignatura del sistema curricular que se inicia desde los primeros años de escolaridad y llega al nivel de educación superior aún con grandes dificultades en la realización de las operaciones básicas en la disciplina.

\section{Marco metodológico.}

Considerando que este estudio pretende mejorar el desarrollo del proceso de enseñanza aprendizaje de la matemática en la institución LB "José Véliz" mediante talleres de formación en técnicas de superaprendizaje para el docente, el mismo se enmarca dentro del paradigma Crítico - Social, en el tipo Investigación Cualitativa, específicamente en la modalidad Acción Participante, considerando sus 4 fases (observación participante, investigación participativa, la acción participativa y la evaluación) con la intención de transformar la realidad educativa y promover mejoras en el proceso de enseñanza aprendizaje de una disciplina tan esencial en el campo de la educación secundaria.

En ese sentido, la investigación acción en este estudio, consiste en comprobar en el ambiente de clases, la aplicación de técnicas innovadoras de aprendizaje acelerado que sirvan de impulso a las transformaciones en la praxis educativa que se desarrolla en el LB "José Véliz", en el área de matemática. Los procedimientos para seguir en este proceso de investigación, se sustentan en los pasos propuestos por Lewin (1946), (citado en Latorre 2007), para la realización de una investigación acción. El autor describió este tipo de investigación como ciclos de acción reflexiva compuestos en una espiral de pasos que son: planificación, implementación y evaluación del resultado de la acción. 
Al respecto, Lewin (1946), (citado en Latorre 2007), expresa que en este tipo de estudio se parte de una idea central sobre algún tema de interés para los involucrados, la cual es desglosada a través de un plan de acción. Seguidamente, se hace una revisión preliminar del plan elaborado, analizando sus fortalezas y debilidades, para luego ejecutar el primer paso de acción planificada y evaluar los resultados. Esto permitirá reflexionar sobre los logros y dificultades en los procedimientos desarrollados para de esta manera planificar el segundo paso de la acción considerando la valoración del primero.

En referencia a lo descrito, para lograr el potencial total de mejora y cambio en este proceso de investigación, se plantearon seis ciclos de acción, los cuales serán sometidos constantemente a los procesos de revisión que se requieran para realizar los ajustes o reimpulsar el plan general de la acción, que será reestructurado en la medida en que se conciba la transformación de la realidad estudiada.

Por otro lado, en cuanto a los instrumentos de recolección de datos según Ander-Egg (2011: 21), son los medios que utiliza el investigador para medir el comportamiento o atributos de las variables. Entre estos se pueden mencionar: los cuestionarios, entrevistas, grupos y asambleas para discusión, triangulaciones, observaciones, escalas de clasificación, pruebas estandarizadas, entre otros.

En el estudio que se realizó en la institución LB "José Véliz", la información se recabó mediante la aplicación de observación participante, la entrevista, la lluvia de ideas, el registro anecdótico, grupos de discusión y la triangulación de la información para establecer un diagnóstico mucho más preciso de los sujetos y el campo de estudio.

De la misma manera, para el análisis de los datos, se definió la necesidad prioritaria seleccionada por los participantes, por medio del gráfico de tortas visualizando la información, para posteriormente ser analizada, simplificada e interpretada y mediante la técnica de la espina de pescado, se 
resumieron los problemas considerando las causas y efectos para su posterior resolución.

\section{Resultados y análisis de la información}

Enumerados y jerarquizados los problemas, se procedió a la elaboración de un diagrama de tortas para presentar la información del análisis situacional del plantel, quedando enumerados de la siguiente manera:

Figura 1. Problemas que inciden en el aprendizaje de los estudiantes del LB "José Véliz".

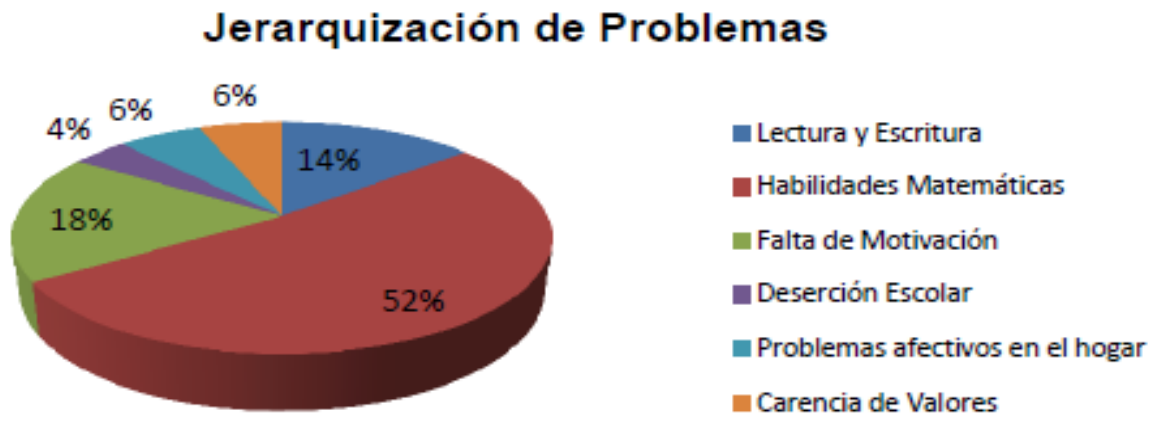

Fuente: Páez (2013).

Para desarrollar este tipo de investigación acción participante, fue necesario propiciar un proceso de interacción o acercamiento con los actores del estudio, por lo cual se diseñó el siguiente plan de acción:

Tabla 1. Plan de acción para sensibilizar a los participantes.

\begin{tabular}{|c|c|c|c|c|}
\hline ACTIVIDAD & ACTORES & FECHA & RECURSOS & RESULTADOS \\
\hline $\begin{array}{l}\text { Discusión grupal sobre los } \\
\text { problemas que inciden en el } \\
\text { proceso de enseñanza } \\
\text { aprendizaje. } \\
\text { (Cognitivos y Emocionales) }\end{array}$ & $\begin{array}{c}\text { Investigador, } \\
\text { Personal Directivo } \\
\text { y Docentes }\end{array}$ & $\begin{array}{c}13 \text { de } \\
\text { Noviembre de } \\
2013\end{array}$ & $\begin{array}{c}\text { Pizarra, marcadores } \\
\text { acrílicos, lectura reflexiva, } \\
\text { papel Bond, lápiz, vídeo } \\
\text { Beams. }\end{array}$ & $\begin{array}{l}\text { Diagnóstico grupal. } \\
\text { Trabajo en equipo. }\end{array}$ \\
\hline $\begin{array}{c}\text { Dinámica grupal ¿Qué piensas } \\
\text { de mí? }\end{array}$ & $\begin{array}{l}\text { Investigador. } \\
\text { Personal directivo } \\
\text { y docente. }\end{array}$ & $\begin{array}{l}23 \text { de Enero } \\
\text { de } 2014\end{array}$ & $\begin{array}{l}\text { Papel, lápiz, lectura } \\
\text { reflexiva. }\end{array}$ & $\begin{array}{c}\text { Clima de armonía en el } \\
\text { equipo. } \\
\text { Participación. }\end{array}$ \\
\hline $\begin{array}{c}\text { Juego de voleibol entre } \\
\text { profesores. }\end{array}$ & $\begin{array}{l}\text { Investigador y } \\
\text { docentes. }\end{array}$ & $\begin{array}{l}7 \text { de Febrero } \\
\text { de } 2014\end{array}$ & Malla, balón, pito. & $\begin{array}{l}\text { Compañerismo. } \\
\text { Participación. }\end{array}$ \\
\hline
\end{tabular}

Fuente: Páez (2013). 
Asimismo, luego de un exitoso proceso de sensibilización se programó un conjunto de talleres para formar al docente en técnicas de superaprendizaje, con el propósito de abordar el problema de la falta de estrategias innovadoras y orientadoras para la enseñanza de la matemática, así como también las deficiencias que existen en el dominio de la asignatura, para lo cual se realizó un plan general donde se programaron un conjunto de talleres que permitieran el mejoramiento de las condiciones del clima desarrollado en el ambiente de clase y la estimulación a plenitud de los canales sensoriales de modo que predispongan al estudiante a un aprendizaje acelerado de la asignatura, dicho plan fue desarrollado de la siguiente manera:

Tabla 2. Plan General de Acción.

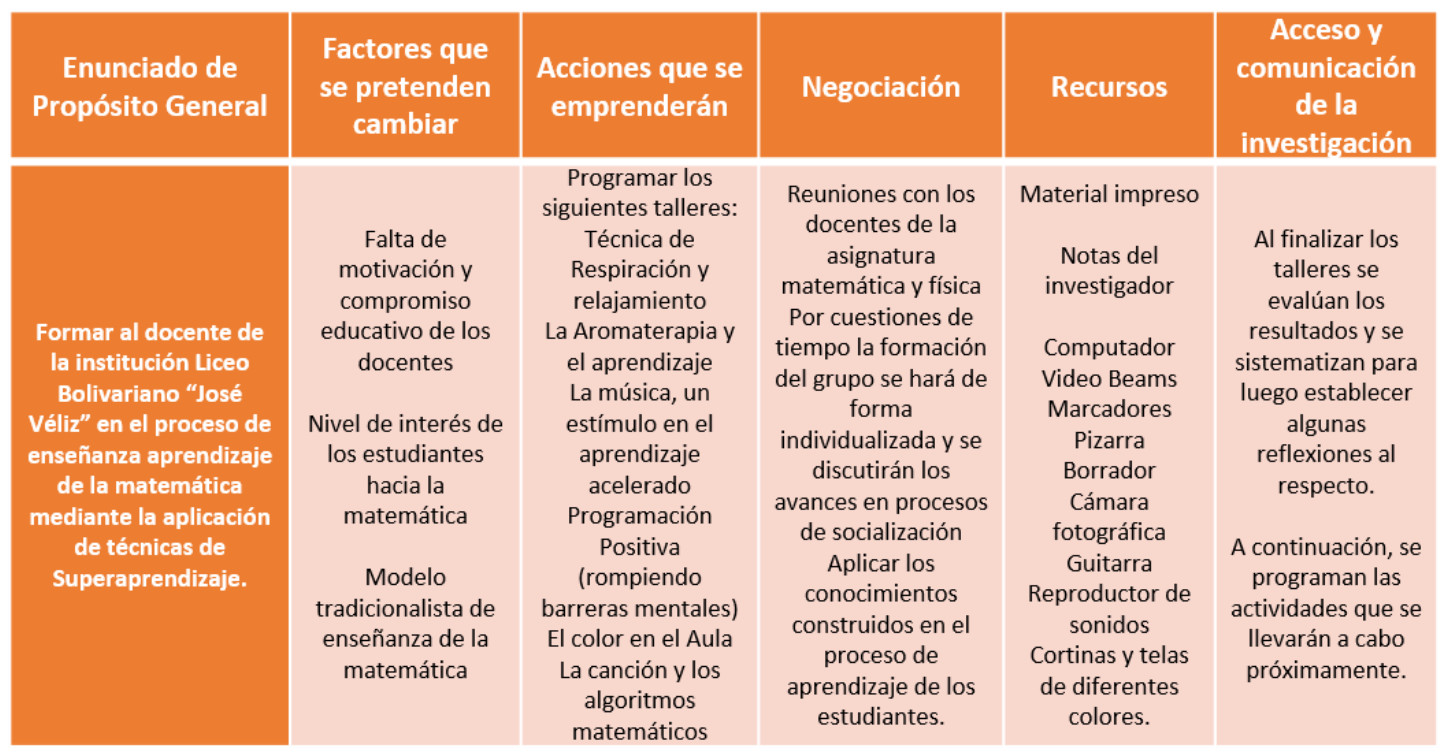

Fuente: Páez (2013).

\section{Conclusiones de la investigación}

En cuanto a las técnicas de respiración y relajación, se propuso la necesidad de hacer uso de las mismas no sólo en el inicio o desarrollo de una clase sino básicamente en los momentos de evaluación, puesto que son los 
instantes en los cuales los estudiantes sienten mayor presión para la toma de decisiones adecuadas en la elección de algún método de resolución de problemas estableciendo, incluso, barreras que dificulten el uso del pensamiento lógico a su mayor capacidad.

Haciendo referencia a las técnicas de aromaterapia, para mejorar las condiciones de aprendizaje de los estudiantes, los participantes valoraron la importancia de la creatividad y la improvisación en ciertas circunstancias especiales y su vez propusieron el uso algunos aromas en situaciones de aprendizaje específicas en la asignatura de matemática en el LB "José Véliz", tales como lo son: el jazmín, el sándalo y la bergamota o lima cuando se trate de evaluaciones $u$ otras actividades que involucren mucha presión o estrés, y el romero para usarlo en algunas clases donde se tengan que explicar ejercicios demostrativos.

En relación con las aplicaciones de la música en el aprendizaje de la matemática, los participantes coincidieron que para fomentar la atención y la concentración en los estudiantes del LB "José Véliz" por las actividades académicas en el área, la música utilizada serán algunas de Mozart tales como la sonata para dos pianos en re mayor y los conciertos para violín 3 y 4 , por los efectos producidos en ellos en el taller desarrollado.

En cuanto a la programación positiva, la mayoría de los participantes valoraron la relevancia que tiene el conocimiento de características sencillas y fáciles de detectar que definen el tipo de estilo de aprendizaje de sus educandos, puesto que al comprender los canales de percepción de las mayorías se pueden aplicar estrategias bien definidas para alcanzar con mayor eficiencia los propósitos establecidos.

Asimismo, los participantes expresaron que en el aula de clases, y sobre todo en la asignatura de matemática, el docente debe eliminar de su vocabulario palabras que usa a menudo y que incitan a lo negativo con frases tales como "Esta materia no es fácil", "Para que me aprueben tendrán que 
estudiar bastante", "Me pasarán como tres o cuatro", "Son muchos los que veré en fase de revisión" y otras que sólo crean barreras mentales a pesar de que las mismas se digan en maneras de bromas y juegos.

Con respecto al uso del color en el aula, los docentes en su mayoría opinaron que los colores ideales en las aulas del LB "José Véliz" deben ser los fríos debido a la situación de las condiciones ambientales del plantel y por las altas temperaturas de la zona, por lo tanto lo que puede permitir la promoción del aprendizaje de la matemática, será una combinación entre azul claro que inspira tranquilidad, con ciertos toques de naranja, que estimula la actividad cerebral, incluyendo algunas franjas de azul rey que impulsan el pensamiento lógico, para lo cual se desarrolló una jornada de pintura en el plantel con la dotación de las mismas por entes gubernamentales representantes del estado Lara.

En lo que se refiere al uso de la canción en el proceso de enseñanza aprendizaje de la matemática en el LB "José Véliz", los participantes, plantearon la posibilidad de introducir en cada institución educativa un especialista en el área de música que cumpla con el perfil adecuadamente, teniendo habilidades para tocar más de dos instrumentos, cantar, componer canciones, y poesías, tener dominio de la vocalización, preparado en lectura y escritura y aplicar adecuadamente las técnicas de respiración, de modo que sirva de apoyo al docente de matemática, y otras áreas críticas, en la implementación de algunas estrategias.

Por último, producto de los talleres desarrollados y como complemento al trabajo investigativo realizado, se desarrolló una estrategia para presentar de manera ordenada el proceso de formación del docente en el abordaje de técnicas de superaprendizaje que permitan mejorar las condiciones en el ambiente escolar para la enseñanza de la matemática. 


\section{Referencias}

Álamo G., y Moronta D., (2007). Superaprendizaje: Estrategias Estimulantes para Facilitar el Proceso de Pensamiento. Maracaibo, Venezuela. Revista ORBIS / Ciencias Humanas. Año 3/ № 7.

Ander-Egg E., (2011). Repensando la Investigación-Acción-Participativa. Santa Fe. Colombia. Grupo editorial Lumen Humanitas. 4ta. edición.

Armstrong, T., (2006). Inteligencias múltiples en el aula. Barcelona. España.

Editorial Paidós.

Bronzina L., y otros (2009). Aportes para la Enseñanza de la Matemática.

Laboratorio Latinoamericano de Evaluación de la Calidad de la

Educación (LLECE). Santiago, Chile. Salesianos Impresiones S.A.

Latorre A., (2007). La Investigación Acción. Conocer y cambiar la práctica educativa. Barcelona, España. Editorial GRAÓ.

López de Ceballos, P., (2008). Un método para la investigación-acción participativa. (4ta. Ed.). Madrid, España: Ed. Popular.

Martínez, M., (2009). Ciencia y arte en la metodología cualitativa. México: Editorial Trillas.

Martínez, M. (2009). La psicología humanista: un nuevo paradigma psicológico. México: Editorial Trillas.

Santaolalla E., (2009). Matemáticas y estilos de aprendizaje. Revista Estilos de Aprendizaje, nro. 4, vol. 4. Universidad Nacional Educación a Distancia - UNED - España. 


\section{Avilner Rafael Páez Pereira \\ e-mail: profavilnerp@gmail.com}

Nacido en Carora, Estado Lara, Venezuela, el 24 de

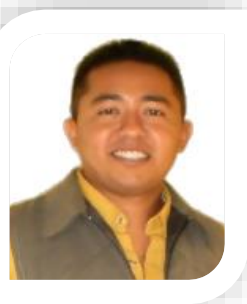

Marzo de 1984. Cursó estudios de pregrado en la Universidad Nacional Experimental "Rafael María Baralt" (UNERMB), obteniendo a los 23 años el título de Lcdo.

Educación Mención Integral graduado con la distinción CUM LAUDE, pero su pasión por las matemáticas lo llevó a cursar estudios de pregrado en esta área en la misma casa de estudios en paralelo con la maestría en Docencia para la Educación Superior en diferentes sedes, para obtener a los 28 años los títulos de Lcdo. en Educación Mención Matemática y Física y Magíster Scientiarum en Docencia para la Educación Superior siendo el primer lugar en ambas carreras. Posteriormente realizó el Doctorado en Educación en la Universidad Nacional Experimental "Rafael María Baralt" (UNERMB), estado Zulia en la espera del título hasta la fecha. En la actualidad se desempaña como Docente de aula por horas en el Liceo Bolivariano "José Véliz" y enlace de formación del circuito educativo $N^{\circ} 17$ del municipio Torres, estado Lara con 11 años al servicio en el Ministerio del Poder Popular para la Educación.

El contenido de este manuscrito se difunde bajo una Licencia de Creative Commons ReconocimientoNoComercial-Compartirlgual 4.0 Internacional 